\title{
Oplosmiddelvrye-transformasie van bensielalkohol na bensaldehied oor koper- en sink-gemodifiseerde fosfomolibdeensuur-katalisators by kamertemperatuur
}

Outeurs:

Jhansi Pedada

Holger B Friedrich

Sooboo Singh

\section{Affiliasies:}

Skool vir Chemie en Fisika, Universiteit van KwaZuluNatal, Durban, Suid-Afrika, 4000

Korresponderende outeur: Sooboo Singh

Epos: singhso@ukzn.ac.za

Datums:

Ontvang: $\quad 19 / 02 / 21$

Aanvaar: $\quad 28 / 04 / 21$

Gepubliseer: 06/07/21

Hoe om hierdie artikel aan te haal:

Jhansi Pedada, Holger B

Friedrich, Sooboo Singh,

Oplosmiddelvrye-

transformasie van

bensielalkohol na ben-

saldehied oor koper- en

sink-gemodifiseerde

fosfomolibdeensuur-

katalisators by kamer-

temperatuur, Suid-

Afrikaanse Tydskrif vir

Natuurwetenskap en

Tegnologie 40(1) (2021).

https://doi.org/10.36303/

SATNT.2021.40.1.842

An English copy of this paper is available online at http://www.satnt.ac.za/ index.php/satnt/article/ view/842

\section{Kopiereg:}

C) 2021. Authors

Licensee: Die Suid-

Afrikaanse Akademie vir

Wetenskap en Kuns.

Hierdie werk is onder

die Creative Commons

Attribution License

gelisensieer.
Koper- en sinkuitgeruilde heteropolisuurkatalisators is gesintetiseer en gekarakteriseer deur verskeie tegnieke soos X-straaldiffraksie (XSD), infrarooispektroskopie (IR), piridiengeadsorbeerde infrarooispektroskopie, BET-oppervlakanalise, ${ }^{31} \mathrm{P}$ KMR-spektroskopie en Induktief Gekoppelde Plasma Optiese Emissiespektroskopie (IGP-OES) om die fisieschemiese eienskappe daarvan te ondersoek. Poeier-X-straaldiffraktogramme het getoon dat kristalliete van die Keggin-ioon van die heteropolisuur teenwoordig was en dat dit behoue gebly het na metaalmodifikasie. Piridien-geadsorbeerde infrarooispektroskopie het aangedui dat die Brønsted/Lewis-suurplekverhouding toegeneem het in die metaaluitgeruilde fosfomolibdeensuur-katalisators, terwyl daar ' $n$ afname was in hierdie verhouding vir die gemodifiseerde fosfowolframsuurkatalisators. ${ }^{31} \mathrm{P}$ NMR-spektra het getoon dat die metaal suksesvol met protone uitgeruil is en nie geïnkorporeer is in die primêre struktuur van heteropolisuurkatalisator nie; dus is die Keggin-ioon behou. In die oksidasie van bensielalkohol deur metaaluitgeruilde katalisators, het die $\mathrm{Zn}$-uitgeruilde fosfomolibdeensuur-katalisators hoër oksidasie-aktiwiteit getoon wat goed gekorreleer het met die suurheid van die katalisators.

Trefwoorde: PMA, PTA, bensielalkohol, bensaldehied, TBHP

Solvent free transformation of benzyl alcohol to benzaldehyde over copper and zinc modified phosphomolybdic acid catalysts at room temperature: Copper and zinc exchanged heteropolyacid catalysts were synthesised and characterised by various techniques such as X-ray diffraction, infrared spectroscopy, pyridine adsorbed infrared spectroscopy, BET surface studies, ${ }^{31} \mathrm{P}$ NMR spectroscopy and inductively coupled plasma-optical emission spectroscopy to investigate its physico-chemical properties. Powder X-ray diffractograms showed that crystallites of the Keggin ion of the heteropolyacid were present and were retained after metal modification. Pyridine adsorbed infrared indicated that the Brønsted/ Lewis acid site ratio increased in the metal exchanged phosphomolybdic acid catalysts, whereas there was a decrease in this ratio for the modified phosphotungstic acid catalysts. ${ }^{31} \mathrm{P}$ NMR spectra showed that the metal was successfully exchanged with protons and was not incorporated in the primary structure of heteropolyacid catalyst, thus maintaining the Keggin ion. In the oxidation of benzyl alcohol over metal exchanged catalysts, the $\mathrm{Zn}$ exchanged phosphomolybdic acid catalysts showed higher oxidation activity and correlated well with the acidity of the catalysts.

Keywords: PMA, PTA, benzyl alcohol, benzaldehyde, TBHP

\section{Inleiding}

Die omskakeling van bensielalkohol in bensaldehied onder omgewingsvriendelike toestande bly gewild en is 'n uitdaging vir die akademiese sektor en die chemiese industrie as gevolg van die belangrikheid van bensaldehied in basiese en toegepaste navorsing (Fey et al., 2001; Franz et al., 1991; Regen en Koteel, 1977; Sheldon, 1991; Xia et al., 2005). Bensaldehied is 'n veelsydige aromatiese karbonielverbinding wat in die parfuum-, skoonheids- en landbouchemiese nywerhede gebruik word. Verskeie katalitiese materiale is ontwikkel om bensaldehied uit bensielalkohol te produseer deur homogene en heterogene oksidasiekatalise (Bordoloi et al., 2008; Chen et al., 2014; Farsani et al., 2014 Nadealian et al., 2012; Nikbakht et al., 2015; Pathan 
en Patel, 2104; Tundo et al., 2010). By homogene katalise word probleme ondervind met die herwinning van die katalisator en is groot hoeveelhede oplosmiddels nodig wat duur en nie omgewingsvriendelik is nie. As gevolg hiervan het oplosmiddelvrye heterogene katalisators meer aandag gekry weens die maklike herwinning en herwinbaarheid. Van die verskillende materiale wat bestudeer is, het vastesuurkatalitiese materiale, soos heteropolisure, gewild geword weens die bifunksionele suur- en redokskarakter daarvan (Choi et al., 2014; Choudhary et al., 2009; Fan et al., 2016; Farhadi and Zaidi, 2009; Haimov en Neumann, 2002; Hasannia en Yadollahi , 2015; Ma et al., 2017; Palermo et al., 2014; Park et al., 2015; Zhao et al., 2013). Daarbenewens is dit 'n groot voordeel dat die suur-basis- en redokseienskappe ingestel kan word deur eenvoudige uitruil van protone met metale.

$\mathrm{Zn}$ - en $\mathrm{Cu}$ - uitgeruilde heteropolisuurkatalisators is bestudeer met betrekking tot verskeie reaksies, soos die Knoevenagel-kondensasiereaksie, oksidasiereaksies en suurgekataliseerde organiese transformasies (Jagadeeswaraiah et al., 2014; Patil et al., 2014; Rao et al., 2012; Pope, 1991; Viswanadham et al., 2016). Khabazzadeh et al. (2009) het $\mathrm{Cu}_{1.5} \mathrm{PMo}_{12} \mathrm{O}_{40}$ en $\mathrm{Cu}_{1.5} \mathrm{PW}_{12} \mathrm{O}_{40}$ herbruikbare en doeltreffende katalisators ontwikkel vir die bereiding van 1-amidoalkiel-2-naftole, terwyl Patil et al. (2014) die laetemperatuur-aktivering van metaan oor 'n Zn-uitgeruilde heteropolisuur bestudeer het wat gelei het tot die selektiewe vorming van metanol en asynsuur tydens die reaksie. Jagadeeswaraiah et al. (2014) het aangetoon dat die toevoeging van $\mathrm{Zn}^{2+}$ ione in die sekondêre struktuur van heteropolisure doeltreffend was tydens gliserolkarbonaatsintese uit gliserol en ureum, terwyl Rao et al. (2012), wat niobia ondersteun op Zn-uitgeruilde fosfowolframsuur berei het, opgemerk het dat die katalisators goeie aktiwiteit getoon het tydens die omskakeling van furfurielalkohol na butiellevulinaat en dit in verband gebring het met die suurposisies van katalisators. In 'n studie wat gedoen is met behulp van Cu-fosfomolibdeensuur (Cu-PMA), het die outeurs bevind dat die $\mathrm{Cu}$-katalisator beter katalitiese werkverrigting toon as die blanko, dit wil sê fosfomolibdeensuur (PMA). Hierdie beduidende toename in die aktiwiteit van die katalisator is gekorreleer met sy strukturele en suureienskappe.

Die doel van die huidige ondersoek was om verskeie Cuen Zn-uitgeruilde heteropolisuurkatalisators te ontwikkel vir die oksidasie van bensielalkohol en om die invloed van verskillende reaksieparameters soos reaksietemperatuur, katalisatorbelading, molverhouding van reaktant tot oksidant, verskillende oksidante, "tyd-in-stroom" en regenerasie vir hierdie reaksie te verstaan.

\section{Eksperimenteel Katalisatorvoorbereiding}

$\mathrm{H}_{3} \mathrm{PMo}_{12} \mathrm{O}_{40}$ en $\mathrm{H}_{3} \mathrm{PW}_{12} \mathrm{O}_{40}$, sinksulfaathidraat, kopersulfaathidraat en bariumhidroksied is van Sigma-Aldrich (RSA) verkry. Die Zn- en Cu-uitgeruilde katalisators is volgens 'n metode wat vroeër beskryf is (Pope, 1991), gesintetiseer. In 'n tipiese sintese is $0.10 \mathrm{M}$ bariumhidroksied by ' $\mathrm{n}$ waterige mengsel van $0.22 \mathrm{M}$ fosfomolibdeensuur in $5 \mathrm{~mL}$ gedistilleerde water gevoeg. Daarna is $0.15 \mathrm{M}$ van $\mathrm{CuSO}_{4} \cdot 5 \mathrm{H}_{2} \mathrm{O}$ bygevoeg om barium met koper te vervang deur die neerslag van die barium as $\mathrm{BaSO}_{4}$. Die $\mathrm{Cu}_{1.5} \mathrm{PMo}_{12} \mathrm{O}_{40}$ vastestof is deur sentrifugering herwin en vir 10 uur by 100 ${ }^{\circ} \mathrm{C}$ in 'n oond gedroog. Dié katalisator is aangedui as $\mathrm{Cu}-$ PMA. Op dieselfde wyse is die ander katalisators, naamlik $\mathrm{Cu}_{1.5} \mathrm{PW}_{12} \mathrm{O}_{40}, \mathrm{Zn}_{1.5} \mathrm{PMo}_{12} \mathrm{O}_{40}$ en $\mathrm{Zn}_{1.5} \mathrm{PW}_{12} \mathrm{O}_{40^{\prime}}$ onderskeidelik aangedui as Cu-PTA, Zn-PMA en Zn-PTA, gesintetiseer.

\section{Katalisatorkarakterisering}

Poeier-X-straaldiffraksiepatrone van geselekteerde katalisators is verkry met behulp van 'n Bruker D8 Advancediffraktometer met 'n gekoppelde Lynxeye-detektor, wat toegerus is met ' $\mathrm{n} \mathrm{Cu} \mathrm{K}$-stralingsbron $(\lambda=1.5406 \AA)$ by 40 $\mathrm{kV}$ en $30 \mathrm{~mA}$. Die $2 \theta$-metings is in stappe van $0.045^{\circ}$ met ' $\mathrm{n}$ teltyd van $0.5 \mathrm{~s}$ in die gebied $5^{\circ}$ tot $40^{\circ}$ opgeneem. Die oppervlak-area is met behulp van $\mathrm{N}_{2}$-adsorpsie-isoterme by $196{ }^{\circ} \mathrm{C}$ deur die multipunt BET metode bepaal met 0.162 $\mathrm{nm}^{2}$ as die deursnee-area. Voor hierdie eksperimente is die materiale oornag by $200{ }^{\circ} \mathrm{C}$ in 'n heliumvloei met behulp van'n Micrometrics Flow Prep 060 ontgasser ontgas en alle eksperimente is uitgevoer teen 'n relatiewe drukbereik (P/ Po) van 0.05 tot 0.9. Infrarooi (IR) spektra is op 'n Perkin Elmer opgeneem, toegerus met 'n universele diamantATR-monsterbykomstigheid. Die verpoeierde materiaal is op die diamantkristal geplaas en 'n krag van 120 psi is aangelê om behoorlike kontak tussen die materiaal en die kristal te verseker. Die spektra is met behulp van Spectrum 100-sagteware geanaliseer. Ex-situ piridien geadsorbeerde FT-IR eksperimente is uitgevoer op 'n Perkin Elmer ATR spektrometer by kamertemperatuur. Piridienadsorpsieeksperimente is voor analise uitgevoer deur ' $n$ druppel piridien op ' $n$ klein hoeveelheid van die katalisator te plaas, gevolg deur evakuering vir 1 uur in lug om die omkeerbaar geadsorbeerde piridien te verwyder. Die metaalinhoud is bepaal deur IGP-OES-analise met behulp van 'n Perkin Elmer Optima 5300 DV-spektrometer, terwyl ${ }^{31} \mathrm{P}-\mathrm{KMR}$ spektra op 'n Bruker Advance $400 \mathrm{MHz}$-spektrometer opgeneem is, waar chemiese verskuiwings gerapporteer is relatief tot $85 \% \mathrm{H}_{3} \mathrm{PO}_{4}$ in $\mathrm{D}_{2} \mathrm{O}$. Laasgenoemde is gebruik as eksterne standaard by $298 \mathrm{~K}$.

Die oplosmiddelvrye oksidasie van bensielalkohol is in ' $n$ $10 \mathrm{ml}$ rondebolfles by kamertemperatuur bestudeer. In ' $\mathrm{n}$ tipiese eksperiment is $10 \mathrm{mmol}$ bensielalkohol, $10 \mathrm{mmol}$ $70 \%$ TBHP en $0.05 \mathrm{~g}$ van die katalisator onder roering in die fles geplaas. Die reaksie is voortdurend gemonitor deur ' $n$ Perkin Elmer Auto-gaschromatograaf wat toegerus was met 'n vlamionisasiedetektor (GC-VID), en TurboMatrix ${ }^{\mathrm{TM}}$ sagteware is gebruik. Die katalisator is herwin en weer gebruik vir die reaksie deur herhaling van die beskryfde prosedure. 


\section{Resultate en bespreking Karakterisering van katalisators}

X-straaldiffraksieprofiele van die stam (PMA) en die metaal uitgeruilde heteropolisuurkatalisators word in Figuur 1 getoon. Die suiwer fosfomolibdeensuur (PMA), $\mathrm{Cu}-\mathrm{PMA}$ - en $\mathrm{Zn}$-PMA- katalisators vertoon verskillende diffraksiepieke by $2 \theta=8.2^{\circ}, 9.1^{\circ}, 26.5^{\circ}$ en $28.8^{\circ}$ en verteenwoordig kenmerkende diffraksiepieke van die primêre struktuur van 'n Keggin-ioon (Viswanadham et al., 2016). Daarteenoor, in die geval van PTA-, Cu-PTA- en Zn-PTAkatalisators het die kenmerkende Keggin-ioon van die stam PTA egter ongeskonde gebly na belading van $\mathrm{Cu}$ en $\mathrm{Zn}$. Infrarooispektroskopie bevestig ook die primêre struktuur van die Keggin-ioon. Ex-situ piridienstudies is uitgevoer om die relatiewe suurheid van die katalisators te bepaal. Die infrarooibande by 1529-1538, 1483-1487, 1439-1442 cm-1 verteenwoordig onderskeidelik Brønsted (B), Brønsted + Lewis- (B + L) en Lewis (L) suurposisies (Figuur 2).

Die B/L suurposisieverhouding het aansienlik toegeneem vir die metaaluitgeruilde PMA-katalisators, maar het

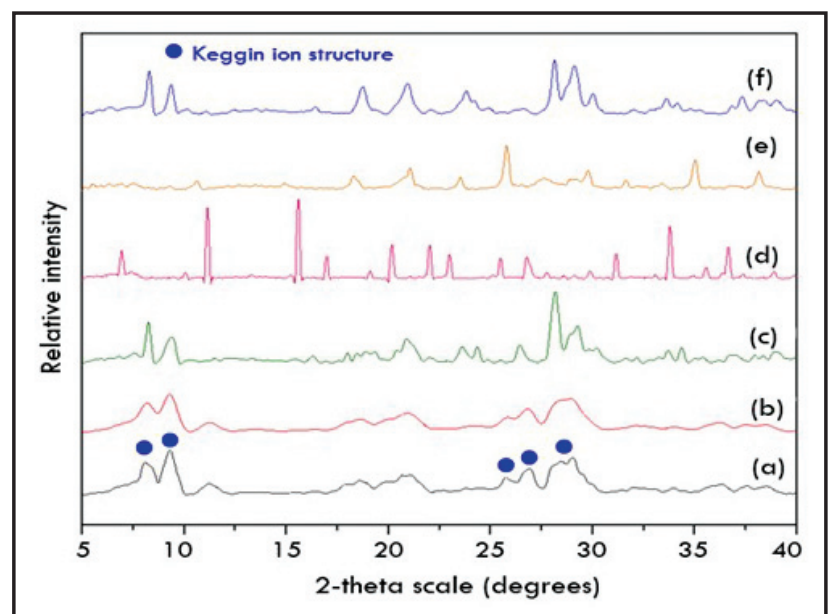

FIGUUR 1: Poeier-XSD-profiel van (a) PMA-, (b) Cu-PMA-, (c) Zn-PMA-, (d) PTA-, (e) Cu-PTA- en (f) Zn-PTA-katalisators.

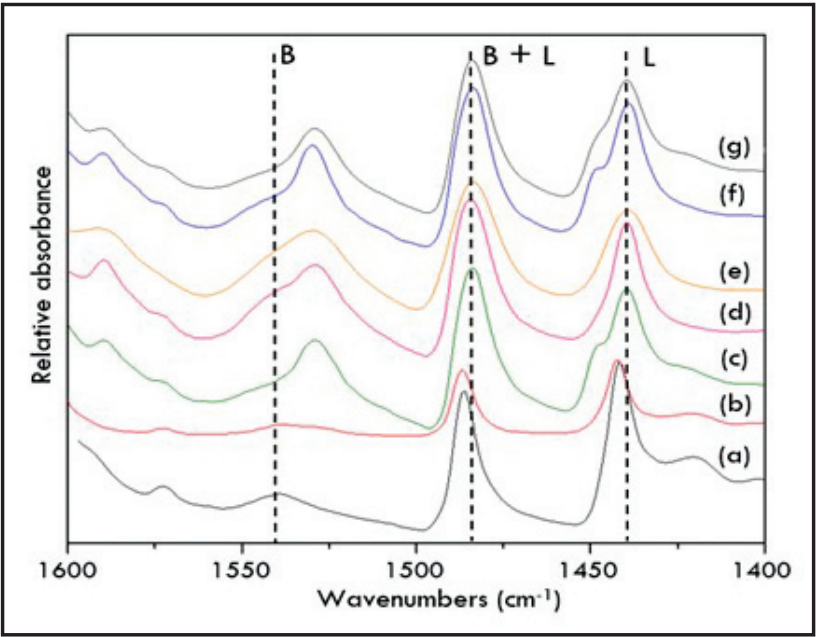

FIGUUR 2: Piridien-vingerafdruk-infrarooispektra van (a) PMA, (b) Cu-PMA (c) Zn-PMA, (d) PTA, (e) Cu-PTA, (f) Zn-PTA en (g) geregenereerde Zn-PMA katalisators $(B=$ Brønsted en $L=$ Lewis). afgeneem in die geval van PTA-katalisators. ${ }^{31} \mathrm{P}$ NMRspektra van die stam, $\mathrm{Cu}$ en $\mathrm{Zn}$ metaaluitgeruilde heteropolisuurkatalisators toon die teenwoordigheid van 'n enkele sein by -3.89 ppm weens die fosforatome in die PMA, terwyl dit by PTA waargeneem is by $-15.20 \mathrm{ppm}$ (Figuur 3).

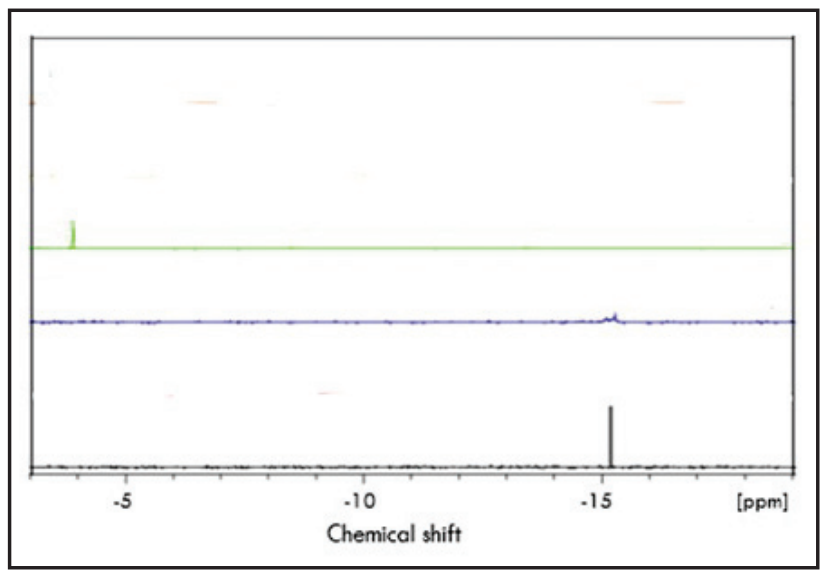

FIGUUR 3: ${ }^{31}$ P NMR spektra van (a) PMA, (b) 0.5CuPTA en (c) PTA

Na die uitruil van die $\mathrm{Cu}$ met $\mathrm{Zn}$ vertoon die katalisators ' $\mathrm{n}$ effense verskuiwing in die piek, maar dit het geen noemenswaardige effek in die fosforomgewing wat gehandhaaf word nie. Hierdie resultate stem goed ooreen met studies wat deur ander navorsers gedoen is (Rao et al., 2012; Viswanadham et al., 2016). IGP-analises en BEToppervlakareas van die metaaluitgeruilde heteropolisuurkatalisatore se resultate word in Tabel 1 getabelleer.

TABEL 1: BET oppervlakarea, elementsamestelling en B/L suurposisies van Cuen Zn- uitgeruilde heteropolisuurkatalisators

\begin{tabular}{l|c|c|c|c}
\hline Katalisator & $\begin{array}{c}\text { Oppervlakte } \\
\left(\mathbf{m}^{2} / \mathbf{g}\right)\end{array}$ & $\begin{array}{c}\mathrm{Cu} / \text { Zn-belading } \\
\text { Bereken. (\%) }\end{array}$ & $\begin{array}{c}\text { Cu/Zn-belading } \\
\text { Expt. (\%) }\end{array}$ & $\begin{array}{c}\text { B/L } \\
\text { verhouding }\end{array}$ \\
\hline PMA & 2.5 & - & - & 0.44 \\
\hline Cu-PMA & 6.9 & 1.5 & 1.42 & 0.46 \\
\hline Zn-PMA & 2.1 & 1.5 & 1.46 & 0.59 \\
\hline PTA & 4.5 & - & - & 0.60 \\
\hline Cu-PTA & 7.5 & 1.5 & 1.45 & 0.54 \\
\hline Zn-PTA & 1.8 & 1.5 & 1.49 & 0.55 \\
\hline Zn-PMA (regen.) & 2.0 & 1.5 & 1.44 & 0.58 \\
\hline
\end{tabular}

$B / L=$ Brønsted / Lewis

Hierdie bevindinge toon aan dat die teoretiese metaalbelading van die uitgeruilde katalisators goed ooreenstem met eksperimentele waardes. Die geregenereerde katalisator toon minimale verandering in die metaalinhoud. Daar was 'n toename in die oppervlakarea van koperuitgeruilde katalisators in vergelyking met die oorspronklike, terwyl 'n afname vir die Zn-katalisators waargeneem is; waarskynlik as gevolg van die metaalgrootte.

Die ${ }^{31} \mathrm{P}$ KMR-spektra van die stam, Cu- en Zn-metaaluitgeruilde heteropolisuurkatalisators toon die teenwoordigheid van 'n enkele sein by -3.89 ppm weens die fosforatome in die PMA, terwyl vir PTA dit waargeneem is by -15.20 ppm. Na uitruiling met $\mathrm{Cu}$ en $\mathrm{Zn}$ vertoon die 
katalisators 'n geringe skuif in die piek, wat geen effek op die fosforomgewing het, wat behoue bly. Hierdie resultate kom goed ooreen met studies van ander navorsers (Rao et al., 2012; Viswanadham et al., 2016).

\section{Katalitiese toetsing}

Die katalitiese eienskappe is bestudeer met inagneming van verskillende reaksieparameters, soos die aard van die katalisator, die katalisatorbelading, TBHP*-konsentrasie, die tipe oksidant, konsentrasie van die reaktant en die reaksietemperatuur. "Tyd-in-stroom" en regenerasie-eksperimente is ook uitgevoer. Die katalitiese oksidasie van bensielalkohol is uitgevoer oor die $\mathrm{Cu}$ - en $\mathrm{Zn}$-uitgeruilde heteropolisuurkatalisators onder 'n stikstofatmosfeer. Die resultate word in Tabel 2 weergegee. $\left(\mathrm{TBHP}^{*}=\right.$ tersbutielhidroperoksied)

Blanko eksperimente wat onder soortgelyke reaksietoestande uitgevoer is, het 'n bensielalkoholomskakeling van $8,0 \%$ getoon met hoë selektiwiteit teenoor bensaldehied. Die stam-heteropolisure, fosfomolibdeensuur het 'n $14.5 \%$ omskakeling getoon, terwyl fosfotungstensuur 'n $13.5 \%$ omskakeling getoon het. In beide gevalle was bensaldehied die belangrikste produk, wat met baie hoë selektiwiteit verkry is. Onder die $\mathrm{Cu}$ - en $\mathrm{Zn}$-uitgeruilde heteropolisuurkatalisators het Zn-PMA katalisators effens beter gevaar in vergelyking met stam- en ander metaaluitgeruilde heteropolisuurkatalisators; waarskynlik as gevolg van 'n effens hoër B/L-suurposisieverhouding.

Die katalitiese eienskappe van verskillende hoeveelhede $\mathrm{Zn}$-PMA-katalisators is bestudeer en die resultate word in Tabel 3 weergegee.

TABEL 2: Katalitiese resultate van die oksidasie van bensielalkohol oor stamen metaal- uitgeruilde heteropolisuurkatalisators

\begin{tabular}{l|c|c|c|c}
\hline \multirow{2}{*}{ Katalisator } & \multirow{2}{*}{ Omskakeling (\%) } & \multicolumn{2}{|c|}{ Selektiwiteit (\%) } & \multirow{2}{*}{ TON } \\
\cline { 3 - 4 } & & Bensaldehied & Bensoësuur & \\
\hline Blanko & 8 & 94 & 6 & - \\
\hline PMA & 15 & 96 & 4 & 30 \\
\hline Cu-PMA & 23 & 95 & 5 & 48 \\
\hline Zn-PMA & 25 & 85 & 15 & 53 \\
\hline PTA & 14 & 98 & 2 & 28 \\
\hline Cu-PTA & 22 & 96 & 4 & 46 \\
\hline Zn-PTA & 24 & 94 & 6 & 50 \\
\hline
\end{tabular}

Reaksietoestande: bensielalkohol (10 mmol), TBHP (10 mmol), katalisator $(0,1$ g), reaksietemperatuur $=$ kamertemperatuur, reaksietyd $=24$ uur, $\mathrm{TON}=$ aantal mol produkte/aantal mol katalisator. (Eksperimente in drievoud uitgevoer).

TABEL 3: Katalitiese resultate van bensielalkoholoksidasie oor verskillende katalitiese hoeveelhede $\mathrm{Zn}$-uitgeruilde PMA katalisators.

\begin{tabular}{l|c|c|c}
\hline \multirow{2}{*}{$\begin{array}{l}\text { Katalisator } \\
\text { hoeveelheid }(\mathrm{g})\end{array}$} & \multirow{2}{*}{ Omskakeling (\%) } & \multicolumn{2}{|c}{ Selektiwiteit (\%) } \\
\cline { 3 - 4 } & & Bensaldehied & Bensoësuur \\
\hline 0,025 & 23 & 76 & 24 \\
\hline 0,05 & 40 & 83 & 17 \\
\hline 0.1 & 25 & 85 & 15 \\
\hline 0.2 & 26 & 86 & 14 \\
\hline
\end{tabular}

Reaksietoestande: bensielalkohol (10 mmol), TBHP $(10 \mathrm{mmol})$, reaksietemperatuur $=$ kamertemperatuur, reaksietyd $=24$ uur. (Eksperimente in drievoud uitgevoer).
Hierdie bevindings dui aan dat die katalitiese aktiwiteit toegeneem het namate die belading van 0.025 tot $0.05 \mathrm{~g}$ toegeneem het. Katalisators met hoër beladings het gelei tot 'n effense toename in die katalitiese aktiwiteit. Die optimale katalisatorhoeveelheid was $0.05 \mathrm{~g}$ en is gebruik in daaropvolgende studies met ander parameters. 'n Toename in die TBHP-konsentrasie het gelei tot ' $n$ toename in die oksidasie-aktiwiteit, maar 'n afname in die selektiwiteit teenoor bensaldehied. Daar is tot die gevolgtrekking gekom dat die optimale waarde van die TBHP $10 \mathrm{mmol}$ TBHP was deur inagneming van sowel omskakeling as die selektiwiteit teenoor bensaldehied (Tabel 4).

Die oksidasievermoë van verskillende oksidante, soos lug, $\mathrm{H} 2 \mathrm{O} 2$ en TBHP vir bensielalkohol-oksidasie is beoordeel oor Zn-PMA katalisators (Tabel 5).

Hierdie bevindings dui aan dat TBHP die doeltreffendste was tydens die oksidasiereaksie. Lug was egter selektiewer, hoewel die aktiwiteit relatief swak was by kamertemperatuur. Die effek van die bensielalkoholkonsentrasie op Zn-PMA-katalisators is onder dieselfde reaksietoestande bestudeer en die resultate word in Tabel 6 weergegee. Die omskakeling was die hoogste vir 'n $2 \%$-voermateriaal, maar die selektiwiteit teenoor bensaldehied was laag en meer bensoësuur is waargeneem.

TABEL 4: Katalitiese resultate van bensielalkohol-oksidasie met verskillende hoeveelhede TBHP oor Zn-uitgeruilde PMA-katalisators.

\begin{tabular}{l|c|c|c}
\hline \multirow{2}{*}{ TBHP (mmol) } & \multirow{2}{*}{ Omskakeling (\%) } & \multicolumn{2}{|c}{ Selektiwiteit (\%) } \\
\cline { 3 - 4 } & & Bensaldehied & Bensoësuur \\
\hline 5 & 22 & 98.0 & 2.0 \\
\hline 10 & 40 & 83.5 & 16.5 \\
\hline 20 & 47 & 76.0 & 24.0 \\
\hline 30 & 50 & 74.0 & 26.0 \\
\hline
\end{tabular}

Reaksietoestande: bensielalkohol (10 mmol), TBHP (5-30 mmol), katalisator $(0,05 \mathrm{~g})$, reaksietemperatuur $=$ kamertemperatuur, reaksietyd $=24$ uur. (Eksperimente in drievoud uitgevoer).

TABEL 5: Katalitiese resultate van bensielalkoholoksidasie deur verskillende oksidante oor Zn uitgeruilde PMA katalisators te gebruik.

\begin{tabular}{l|c|c|c}
\hline \multirow{2}{*}{ Oksidant } & \multirow{2}{*}{ Omskakeling (\%) } & \multicolumn{2}{|c}{ Selektiwiteit (\%) } \\
\cline { 3 - 4 } & & Bensaldehied & Bensoësuur \\
\hline Lug & 2 & 94.5 & 5.5 \\
\hline $\mathrm{H}_{2} \mathrm{O}_{2}$ & 6 & 58.5 & 41.5 \\
\hline TBHP & 40 & 83.5 & 16.5 \\
\hline
\end{tabular}

Reaksietoestande: bensielalkohol $(10 \mathrm{mmol})$, katalisator $(0,05 \mathrm{~g})$, reaksietemperatuur $=$ kamertemperatuur, reaksietyd $=24$ uur. (Eksperimente in drievoud gedoen).

TABEL 6: Katalitiese oksidasieresultate met verskillende konsentrasies bensielalkohol oor Zn-uitgeruilde PMA-katalisators.

\begin{tabular}{l|c|c|c}
\hline \multirow{2}{*}{$\begin{array}{l}\text { Bensielalkohol } \\
\text { (mmol) }\end{array}$} & \multirow{2}{*}{ Omskakeling (\%) } & \multicolumn{2}{|c}{ Selektiwiteit (\%) } \\
\cline { 3 - 4 } & & Bensaldehied & Bensoësuur \\
\hline 2 & 75 & 76.0 & 24.0 \\
\hline 4 & 58 & 79.0 & 21.0 \\
\hline 8 & 47 & 80.0 & 20.0 \\
\hline 10 & 40 & 83.5 & 16.5 \\
\hline
\end{tabular}

Reaksietoestande: TBHP $(10 \mathrm{mmol})$, katalisator $(0,05 \mathrm{~g})$, reaksietemperatuur $=$ kamertemperatuur, reaksietyd $=24$ uur. (Eksperimente in drievoud uitgevoer). 
Daar was ' $n$ afname in omskakeling by hoër konsentrasies bensiealkohol; waarskynlik as gevolg van verdunning. Die effek van reaksietyd en herwinbaarheid van die katalisator is bestudeer en die resultate word onderskeidelik in Figuur $4 \mathrm{~A}$ en $4 \mathrm{~B}$ getoon. Die figuur toon aan dat die aktiwiteit toegeneem met ' $n$ toename in reaksietyd, maar daar was 'n afname in die selektiwiteit teenoor bensaldehied, waarskynlik as gevolg van oormatige oksidasie na bensoësuur, wat 'n relatiewe toename oor tyd getoon het. Nadat dit weer geregenereer is, is die Zn-PMA-katalisator aan verdere studies onderwerp. Daar is 'n effense afname in die aktiwiteit oor drie siklusse, wat ooreenstem met die suurheid van gebruikte katalisators. Daar is ook 'n minimale verandering in die $\mathrm{Zn}$-inhoud na regenerasie.

\section{Gevolgtrekking}

Zn-uitgeruilde heteropolisuur-katalisators vertoon 'n hoër oksidasievermoë as Cu-katalisators in die omskakeling van bensielalkohol na bensaldehied, wat verband hou met die suurheid van die katalisators. XRD- en infrarooi-resultate bevestig dat die primêre struktuur van die Keggin-ioon behoue bly, selfs nadat metale in die struktuur opgeneem is, terwyl piridien-geadsorbeerde infrarooispektroskopie getoon het dat die inkorporering van metaal in PMA ' $n$ toename in die B/L-suurposisieverhouding tot gevolg gehad het, terwyl dit afgeneem het vir die metaaluitgeruilde PTA katalisators. 31P KMR spektra toon duidelik dat die
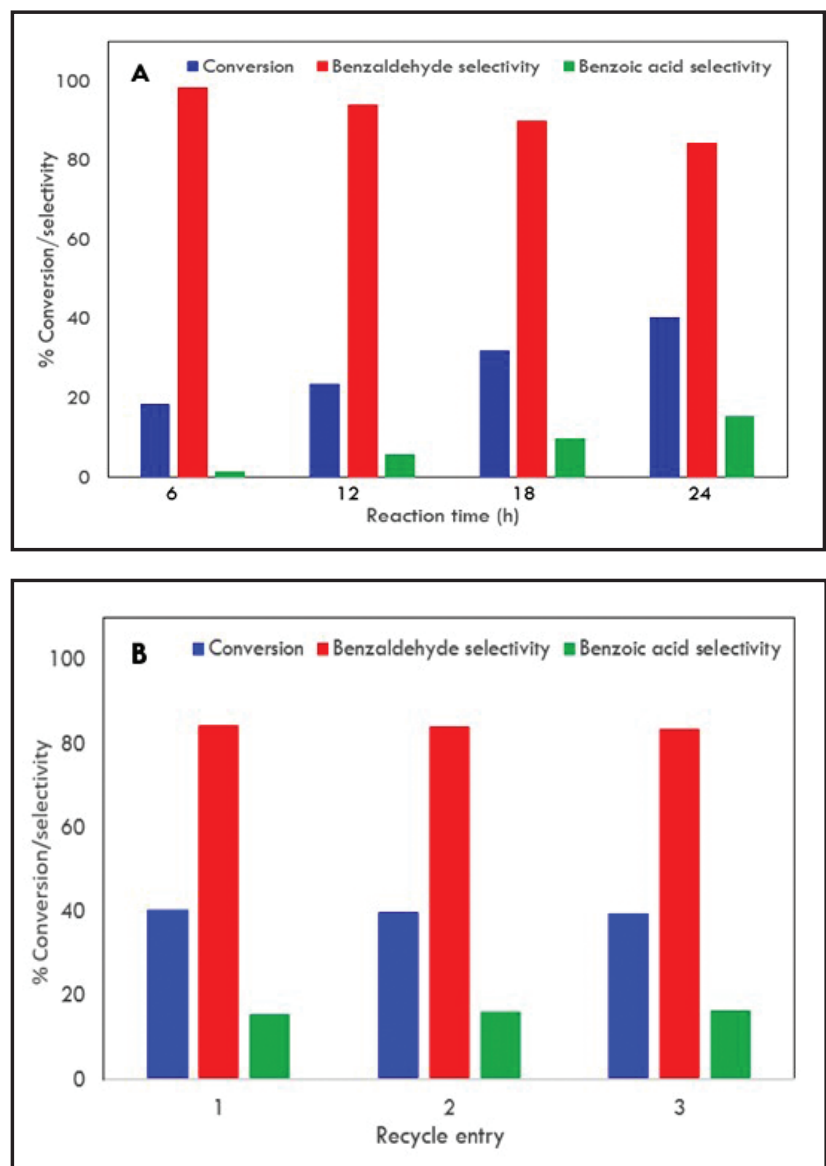

FIGUUR 4: Oksidasie van bensiealkohol oor Zn-PMA-katalisators as ' $n$ funksie van tyd (A) en oor drie siklusse (B). fosforomgewing behoue bly, wat daarop dui dat metaal suksesvol met protone uitgeruil is, maar nie die primêre struktuur van heteropolisuur beïnvloed het nie. Die Znuitgeruilde PMA-katalisators het 'n 97\%-omskakeling van bensielalkohol met 'n 76\%-bensaldehied selektiwiteit opgelewer.

\section{Erkennings}

Die outeurs bedank die Universiteit van KwaZulu-Natal, Westville, vir die voorsiening van navorsingsfasiliteite.

\section{Botsende belange}

Die outeurs verklaar dat daar geen botsing van belange bestaan nie.

\section{ORCID}

Sooboo Singh

https:/ / orcid.org/0000-0001-5765-1013

\section{Verwysings}

Bordoloi A, Sahoo S, Lefebvre F, Halligudi SB. 2008. Heteropoly acid-based supported ionic liquid-phase catalysts for the selective oxidation of alcohols. Journal of Catalysis 259, 232-239. https://doi.org/10.1016/j.jcat.2008.08.010.

Chen G, Zhou Y, Long Z, et al. 2014. Mesoporous Polyoxometalates-based ionic hybrid as a triphasic catalyst for oxidation of benzyl alcohol with $\mathrm{H} 2 \mathrm{O} 2$. ACS Applied Materials and Interfaces 6, 4438-4446. https://doi.org/10.1021/ am5001757.

Choi JH, Kang TH, Bang Y, Song JH, Song IK. 2014. Electrochemical and UVVisible spectroscopy studies of K6As2W18-xMoxO62 ( $x=0-3)$ and WellDawson heteropolyacid catalysts for oxidative dehydrogenation of benzyl alcohol. Catalysis Communications 55, 29-33. https://doi.org/10.1016/j. catcom.2014.06.012.

Choudhary VR, Dumbre DK, Bhargava SK. 2009. Oxidation of benzyl alcohol to benzaldehyde by tert-butyl hydroperoxide over nanogold supported on $\mathrm{TiO}_{2}$ and other transition and rare-earth metal oxides. Industrial Engineering and Chemistry Research 48, 9471-9478. https://doi.org/10.1021/ie801883d.

Fan J, Pu F, Sun M, et al. 2016. Immobilized bis-layered ionic liquids/peroxotungstates as an efficient catalyst for selective oxidation of alcohols in neat water. New Journal of Chemistry 40, 10498-10503. https://doi.org/10.1039/C6NJ01476C

Farhadi S, Zaidi M. 2009. Polyoxometalate-zirconia (POM/ZrO2) nanocomposite prepared by sol-gel process: A green and recyclable photocatalyst for efficient and selective aerobic oxidation of alcohols into aldehydes and ketones. Applied Catalysis A: General 354, 119-126. https://doi.org/10.1016/j. apcata.2008.11.024.

Farsani MR, Jalilian F, Yadollahi B, Rudbari HA. 2014. A comparative study on Keggin and Wells-Dawson Sandwich type polyoxometalates in the oxidation of alcohols with $30 \%$ hydrogen peroxide. Polyhedron 76,102-107. https://doi. org/10.1016/j.poly.2014.03.060.

Fey T, Fischer H, Bachmann S, Albert K, Bolm C. 2001. Silica-supported TEMPO catalysts: synthesis and application in the anelli oxidation of alcohols. Journal of Organic Chemistry 66, 8154-8159. https://doi.org/10.1021/jo010535q.

Franz G, Gerhartz W, Sheldon RA. 1991. Ullmann's Encyclopedia of Industrial Chemistry, fifth ed. VCH Weinheim, Germany.

Haimov A, Neumann R. 2002. Polyethylene glycol as a non-ionic liquid solvent for polyoxometalate catalyzed aerobic oxidation. Chemical Communications 876 877. https://doi.org/10.1039/b200559j.

Hasannia S, Yadollahi B. 2015. Zn-Al LDH nanostructures pillared by Fe substituted Keggin type polyoxometalates: Synthesis, characterization and catalytic effect in green oxidation of alcohols. Polyhedron 99, 260-265. https://doi org/10.1016/j.poly.2015.08.020.

Jagadeeswaraiah K, Kumar CR, Prasad PSS, Lingaiah N. 2014. Incorporation of Zn2+ ions into the secondary structure of heteropoly tungstate: catalytic efficiency for synthesis of glycerol carbonate from glycerol and urea. Catalysis Science and Technology 4, 2969-2977. https://doi.org/10.1039/C4CY00253A.

Khabazzadeh H, Saidi K, Seyedi N. 2009. Cu-exchanged heteropolyacids as efficient and reusable catalysts for preparation of 1-amidoalkyl-2-naphthols. Journal of Chemical Sciences 121, 429-433. https://doi.org/10.1007/s12039-009-00507. 
Ma W, Tong Q, Wang J, et al. 2017. Synthesis and characterization of titanium(IV)/ graphene oxide foam: a sustainable catalyst for the oxidation of benzyl alcohol to benzaldehyde. RSC Advances 7, 6720-6723. https://doi.org/10.1039/ C6RA27690C.

Nadealian Z, Mirkhani V, Yadollahi B, et al. 2012. Selective oxidation of alcohols to aldehydes using inorganic-organic hybrid catalyst based on zinc substituted polyoxometalate and ionic liquid. Journal of Coordination Chemistry 65, 1071 1081. https://doi.org/10.1080/00958972.2011.653965.

Nikbakht E, Yadollahi B, Farsani MR. 2015. Green oxidation of alcohols in water by polyoxometalate nano capsule as catalyst. Inorganic Chemical Communications polyoxometalate nano capsule as catalyst. Inorganic Chemical

Palermo V, Villabrille PI, Gvazquez P, et al. 2013. Role of vanadium and pyridine in heteropoly compounds for selective oxidation of alcohols with hydrogen peroxide. Journal of Chemical Sciences 125, 1375-1383. https://doi. org/10.1007/s12039-013-0523-6.

Park DR, Song JH, Lee SH, et al. 2008. Redox properties of H3PMoxW12-xO40 and H6P2MoxW18-xO62 heteropolyacid catalysts and their catalytic activity for benzyl alcohol oxidation. Applied Catalysis A: General 349, 222-228. https:// doi.org/10.1016/j.apcata.2008.07.042.

PathanS, Patel A. 2014. Keggin type transition metal substituted phosphomolybdates: heterogeneous catalysts for selective aerobic oxidation of alcohols and alkenes under solvent free condition. Catalysis Science and Technology 4, 648-656. under solvent free condition. Catalysis
$\mathrm{https} / / /$ doi.org/10.1039/C3CY00717K.

Patil U, Saih Y, Hamad EA, et al. 2014. Low temperature activation of methane over a zinc-exchanged heteropolyacid as an entry to its selective oxidation to methanol and acetic acid. Chemical Communications 50, 12348-12351. https://doi.org/10.1039/C4CC04950K.

Pope MT. 1991. Heteropoly and Isopoly Oxometalates, thirteenth ed. Springer, Berlin.
Rao KTV, Rao PSN, Nagaraju P, Sai Prasad PS, Lingaiah N. 2012. Room temperature selective oxidation of toluene over vanadium substituted polyoxometalate catalysts. Journal of Molecular Catalysis A: Chemical 358, 67-72.

Regen SL, Koteel C. 1977. Activation through impregnation. Permanganatecoated solid supports. Journal of American Chemical Society 99, 3837-3838. https://doi.org/10.1021/ja00453a053.

Sheldon RA. 1991. Heterogeneous Catalytic Oxidation and Fine Chemicals. Studies in Surface Science and Catalysis 59, 33-54. https://doi.org/10.1016/S01672991(08)61106-4.

Tundo P, Romanelli GP, Vazquez PG, Arico F. 2010. Multiphase oxidation of alcohols and sulfides with hydrogen peroxide catalyzed by heteropolyacids. Catalysis Communications 11, 1181-1184. https://doi.org/10.1016/j. catcom.2010.06.015.

Viswanadham B, Pedada J, Friedrich HB, Singh S. 2016. The Role of Copper Exchanged Phosphomolybdic Acid Catalyst for Knoevenagel Condensation. Catalysis Letters 146, 1470-1477. https://doi.org/10.1007/s10562-016-17777.

Xia QH, Ge HQ, Ye CP, Liu ZM, Su KX. 2005. Advances in Homogeneous and Heterogeneous Catalytic Asymmetric Epoxidation. Chemical Reviews 105, 1603-1662. https://doi.org/10.1021/cr0406458.

Zhao H, Zeng L, Li Y, et al. 2013. Polyoxometalate-based ionic complexes immobilized in mesoporous silica as prepared a one-pot procedure: Efficient and reusable catalysts for $\mathrm{H} 2 \mathrm{O} 2$-mediated alcohol oxidations in aqueous
media. Microporous and Mesoporous Materials 172, 67-76. https://doi. org/10.1016/j.micromeso.2012.12.040. 